\title{
Scapular-focused treatment in patients with shoulder impingement syndrome: a randomised clinical trial
}

\author{
Chris Littlewood
}

Received: 29 January 2013 / Accepted: 30 January 2013 /Published online: 9 February 2013

(C) Clinical Rheumatology 2013

\section{Dear Editor}

I read the paper "Scapular-focused treatment in patients with shoulder impingement syndrome: a randomised clinical trial" by Struyf et al. with great interest and would appreciate further clarification to help better understand the potential implications of the study.

Firstly, the authors describe a statistically significant difference $(p=0.025)$ in favour of the intervention group (scapular-focused treatment) after nine treatment sessions in terms of self-reported shoulder disability. The mean change in the intervention group is 20.9 points on the Shoulder Disability Questionnaire (SDQ), which is regarded as clinically significant, and 2.2 points in the control group (Table 2). Such a small change in the control group is interesting and not in keeping with most, if not all, studies exploring the effects of active interventions in this field. The authors suggest possible reasons for this including potential therapist bias, because both treatments were delivered by the same therapist, but could the timing of the outcomes be another possible reason? I understand that in pragmatic trials of this nature, it is almost impossible to standardise posttreatment outcomes but some descriptive data, e.g. median post-treatment follow-up time and range, would be helpful to the reader to understand whether the measurements were taken at similar times and hence whether the comparisons were fair in this respect.

Secondly, the authors state that after 3 months, both groups improved further. The intervention group reported a mean of 15.6 points on the SDQ and the control group reported 21.7 points, which represents a clinically significant change from baseline for both groups. The paper reports this in terms of no additional improvement in favour of the scapular-focused group. Does this mean that the difference was not statistically significant at this timepoint? If this is the case, considering that the SDQ is the primary outcome, should this have been emphasised more clearly in the paper?

Finally, and related to both of the previous points, was the 3-month follow-up 3 months post-treatment, as stated in the methods sections, or 3 months postrandomisation as seems to be intimated in the results section and in Figure 11? The trial registration page suggests primary outcomes were to be collected after treatment and then at 6 months. Clarity in terms of timing would help the reader better understand the validity of the comparisons that have been made.
C. Littlewood $(\bowtie)$

School of Health \& Related Research, University of Sheffield, Sheffield, UK

e-mail: c.littlewood@sheffield.ac.uk 